\title{
Synchronous X-ray/Optical QPOs from the Black Hole LMXB MAXI J1820+070
}

\author{
Jessymol K. Thomas, ${ }^{1 \star}$ David A. H. Buckley ${ }^{1,2,3}$, Philip A. Charles ${ }^{4}$, John A. Paice ${ }^{4,5}$, \\ Stephen B. Potter ${ }^{1,6}$, James F. Steiner ${ }^{7}$, Jean-Pierre Lasota ${ }^{8,9}$, P. Gandhi ${ }^{4}$, Lian Tao ${ }^{10}$, \\ Xiang $\mathrm{Ma}^{10}$, Yi-Jung Yang ${ }^{10}$, Youli Tuo ${ }^{10}$, Shuang-Nan Zhang ${ }^{10}$ \\ ${ }^{1}$ South African Astronomical Observatory, Observatory Road, Observatory, 7925, Cape Town, South Africa \\ ${ }^{2}$ Department of Astronomy, University of Cape Town, Private Bag X3, Rondebosch 7701, South Africa \\ ${ }^{3}$ Department of Physics, University of the Free State, PO Box 339, Bloemfontein 9300, South Africa \\ ${ }^{4}$ Department of Physics \& Astronomy, University of Southampton, Southampton SO17 1BJ, UK \\ ${ }^{5}$ Inter-University Centre for Astronomy and Astrophysics, Pune, Maharashtra 411007, India \\ ${ }^{6}$ Department of Physics, University of Johannesburg, PO Box 524, Auckland Park 2006, South Africa \\ ${ }^{7}$ Center for Astrophysics | Harvard \& Smithsonian, 60 Garden St. Cambridge, MA 02138, USA \\ ${ }^{8}$ Institut d'Astrophysique de Paris, CNRS et Sorbonne Université, UMR 7095, 98 bis Bd Arago, 75014 Paris, France \\ ${ }^{9}$ Nicolaus Copernicus Astronomical Center, Polish Academy of Sciences, Bartycka 18, 00-716 Warsaw, Poland \\ ${ }^{10}$ Key Laboratory of Particle Astrophysics, Institute of High Energy Physics, Chinese Academy of Sciences, 19B Yuquan Road, Beijing 100049, People's Republic of China
}

Accepted XXX. Received YYY; in original form ZZZ

\begin{abstract}
We present high-speed optical photometry from SAAO and SALT on the black hole LMXB MAXI J1820+070 (ASSASN-18ey), some of it simultaneous with NICER, Swift and Insight-HXMT X-ray coverage. We detect optical Quasi-Periodic Oscillations (QPOs) that move to higher frequencies as the outburst progresses, tracking both the frequency and evolution of similar X-ray QPOs previously reported. Correlated X-ray/optical data reveal a complex pattern of lags, including an anti-correlation and a sub-second lag that evolve over the first few weeks of outburst. They also show correlated components separated by a lag equal to the QPO period roughly centered on zero lag, implying that the inter-band variability is strongly and consistently affected by these QPOs at a constant phase lag of roughly $\pm \pi$. The synchronisation of X-ray and optical QPOs indicates that they must be produced in regions physically very close to each other; we thus propose that they can be explained by a precessing jet model, based on analogies with V404 Cyg and MAXI J1348-630.
\end{abstract}

Key words: black hole physics — Low Mass X-ray Binaries — accretion — accretion discs — stars: individual (MAXI J1820+070)

\section{INTRODUCTION}

A prominent feature of Black Hole X-ray Binaries (BHXBs) in outburst is the presence of quasi-periodic oscillations (QPOs), short time-scale modulations exhibited in the X-ray light-curves. Though the QPOs in BHXB transients have been studied extensively, their origin, evolution and physical nature remain controversial.

Both X-ray and optical QPOs have been seen in the bright BHXB MAXI J1820+070 (ASASSN-18ey) (hereafter J1820), discovered in March 2018 in X-rays by Kawamuro et al. (2018) and then as an optical transient by Denisenko (2018). X-ray QPOs from J1820 have been found by INTEGRAL, who detected a low frequency, $0.04 \pm 0.01$ $\mathrm{Hz}$ QPO with the JEM-X monitor in late March 2018 (Mereminskiy et al. 2018), and AstroSat confirmed an X-ray QPO of $0.0477 \mathrm{~Hz}$

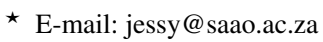

(Mudambi et al. 2020). Also, a QPO at $\sim 0.06 \mathrm{~Hz}$ was reported in April from Swift X-ray power spectra (Yu et al. 2018).

The X-ray QPO properties of J1820 from NICER observations have been presented in detail by Stiele \& Kong (2020), showing how their frequencies evolve smoothly from $\sim 0.03 \mathrm{~Hz}$ on day 10 to $\sim 10$ $\mathrm{Hz}$ at the time of the hard to soft state transition. For convenience we adopt the same time reference as used by Stiele \& Kong (2020) (i.e. day 0 is 2018 Mar 11 0:00 UT = MJD 58188), just before the first triggering of X-rays from J1820 (Kawamuro et al. 2018). Insight-HXMT has detected 1-200 keV X-ray OPQs with frequency gradually evolving from $0.02 \mathrm{~Hz}$ to $0.65 \mathrm{~Hz}$ in the hard and intermediate states (Ma et al. 2021). X-ray QPOs from NuSTAR observations were also reported (Buisson et al. 2019). Furthermore, a QPO of $0.0495 \mathrm{~Hz}$ was reported from optical photometry with the Lijiang $2.4 \mathrm{~m}$ telescope of Yunnan observatories by Yu et al. (2018) on March 30, 2018. The INAF-Astronomical Observatory of Padova also detected a $0.128 \mathrm{~Hz}$ 
optical QPO with IFI+Iqueye and Aqueye on April 18 and 19, and two more QPO-like features $(0.268 \mathrm{~Hz}$ and $0.151 \mathrm{~Hz})$ were detected on June 9-10, using the same instruments (Zampieri et al. 2019).

The number of QPOs detected and their characteristic frequency range depends on the system properties. QPO frequencies $<1 \mathrm{~Hz}$ have been seen in Cyg X-1 (Pottschmidt et al. 2003) and low frequency QPO-like features in the range $\sim 0.03 \mathrm{~Hz}-0.05 \mathrm{~Hz}$ from hard state observations of XTE J1752-223 (Muñoz-Darias et al. 2010). Similar QPOs were also detected from GX 339-4 in 2004 (Motta et al. 2011).

We find low frequency optical QPOs in our J1820 hard state data, which we discuss in Section 3.1 and show in Section 3.2 that they closely track the previously reported X-ray QPOs. We used SALT and the SAAO $1 \mathrm{~m}$ to obtain high-speed photometry at various times in the outburst of J1820. These data were cross-correlated (section 3.3) with simultaneous X-ray observations from NICER, Swift and Insight-HXMT. Our discussion and conclusions are presented in Section 4. For further background information on J1820 together with our long-term variability study see Thomas et al. (2021) (hereafter Paper I).

\section{OBSERVATIONS}

The optical observations of J1820 used here were carried out with the Southern African Large Telescope (SALT) and the South African Astronomical Observatory (SAAO) $1 \mathrm{~m}$ telescope. There are extensive X-ray observations of J1820 throughout the entire outburst with NICER, Swift and Insight-HXMT, some of which have already appeared in the literature, and which we use here to compare with our optical results.

\subsection{SAAO/SHOC}

The high speed photometry of J1820 was performed with the SAAO $1 \mathrm{~m}$ for 20 nights, from 25 March to 29 September 2018. The Sutherland High Speed Optical Camera (SHOC, Coppejans et al. 2013), using a frame-transfer EM-CCD (Andor iXon888), mounted on the $1 \mathrm{~m}$, was used with a clear filter and exposure times of $200 \mathrm{~ms}$. The light curves derived from the images were produced from differential aperture photometry using the SHOC data reduction pipeline ${ }^{1}$. We used GSC 00444-02282 from SIMBAD as a comparison star.

\subsection{SALT- RSS/SALTICAM}

Photometry of J1820 was also performed with SALT during 5 days in June and July 2018. Observations on 13 June and 7 July used SALT's imaging spectrograph (the Robert Stobie Spectrograph, RSS), whereas those on 2 May, 8 July and 10 July employed SALTICAM, which acts as both an acquisition camera and fast science imager.

\subsection{NICER and Insight-HXMT}

We analysed all X-ray observations of J1820 collected by NICER between March and September 2018. NICER data were obtained from the HEASARC data archive and processed via calibration release XTi20200722, screening out any "hot" or problematic detectors.

The Insight-HXMT X-ray light-curve data collected from the Insight-HXMT archive data centre are used here in our optical/X-ray

1 https://shoc.saao.ac.za/Pipeline/SHOCpipeline.pdf comparative studies. The small field-of-view detectors of three payloads (LE: $1-15 \mathrm{keV}, 384 \mathrm{~cm}^{2}$; ME: $5-30 \mathrm{keV}, 952 \mathrm{~cm}^{2}$; HE: $20-$ $250 \mathrm{keV}, 5100 \mathrm{~cm}^{2}$ ) onboard Insight-HXMT were used to generate the light curves in good time intervals (GTIs).

\section{SIMULTANEOUS X-RAY/OPTICAL HIGH SPEED PHOTOMETRY}

The study of simultaneous, fast X-ray and optical variability allows us to probe the emitting regions close to the central black hole in LMXBs. In particular, we focus on the presence of QPOs in J1820 that were detected in both X-ray and optical data.

\subsection{QPOs}

We used the Lomb-Scargle (LS) Periodogram from gatspy.periodic ${ }^{2}$, to perform the period analysis of our optical and $\mathrm{X}$-ray light-curves.

LS power spectra of our SAAO and SALT slotmode data reveal the presence of fast variability and QPOs. We collect together all the QPOs from our SHOC and SALT data in the frequency range $\sim 0.04$ $\mathrm{Hz}$ to $0.3 \mathrm{~Hz}$ in Figure 1, plotted in log-log space to better show the QPOs and for comparison with the X-ray behaviour.

In Figure 1, the SHOC LS power spectra for days 27, 28, 89, and 93 have QPO peaks shown in red, black, green and cyan, respectively, and the blue spectrum is from SALT-RSS on day 95. The optical QPOs observed on days 27, 28, 89, 93, and 95 are at $0.072 \mathrm{~Hz}$, $0.08 \mathrm{~Hz}, 0.278 \mathrm{~Hz}, 0.26 \mathrm{~Hz}$, and $0.27 \mathrm{~Hz}$, respectively, with a 1-sigma uncertainty of $0.01 \mathrm{~Hz}$.

\subsection{Synchronous Optical and X-ray QPOs}

The optical QPOs from our SHOC and SALT observations track the X-ray QPO frequencies from Stiele \& Kong (2020) almost perfectly, as shown by the bars marking the X-ray QPO frequencies in Figure 1 . This immediately indicates that there must be a fundamental connection between their X-ray and optical emitting processes.

The optical and X-ray QPOs from the simultaneous SAAO and Insight-HXMT observations on day 27 were also analysed with LS power spectra. There too we found that the SHOC optical QPO tracks the X-ray QPOs observed from Insight-HXMT, giving the same daily average of $0.072 \mathrm{~Hz}$ in both optical and X-rays. In Figure 2, we show the resulting Insight-HXMT and SHOC power spectra when the data of day 27 are restricted to intervals of exact simultaneity (day 27 in Figure 1 is the average power spectra for the day). This reveals that the optical and X-ray QPO features are more closely aligned (at a frequency of $0.0758 \mathrm{~Hz}$ ) when removing any potential QPO drift between intervals.

The collection in Figure 3 of all the X-ray and optical QPO values demonstrates how well they track each other in frequency throughout the outburst.

\subsection{Simultaneous optical/X-ray cross-correlations}

Optical-X-ray cross-correlations have been carried out for other bright X-ray transients (e.g. Gandhi et al. 2017 for V404 Cyg) and has already been performed on J1820 by Paice et al. (2019, 2021). These

\footnotetext{
2 Gatspy, created by Jake VanderPlas, is a collection of tools for analyzing astronomical time series data in Python (VanderPlas \& Ivezić 2015).
} 


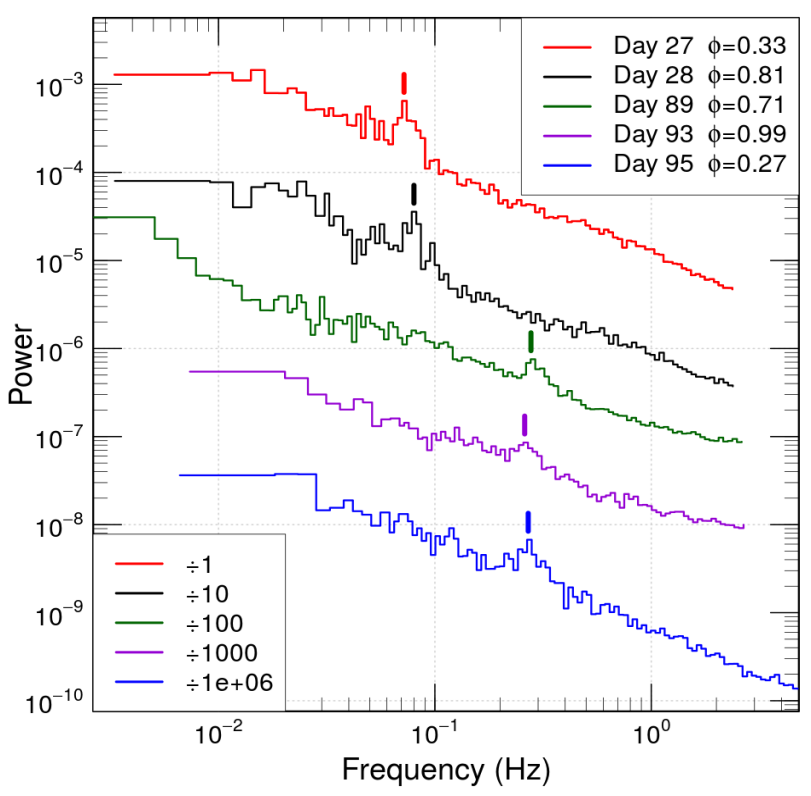

Figure 1. Optical QPOs from SHOC and SALT LS power spectra, identified with colour coding for each day (see box) and arbitrary offsets with power. The vertical bars mark the value of the NICER X-ray QPO fundamental frequencies (Stiele \& Kong 2020), apart from those on days 93 and 95, which are inferred from the upper-harmonic values.

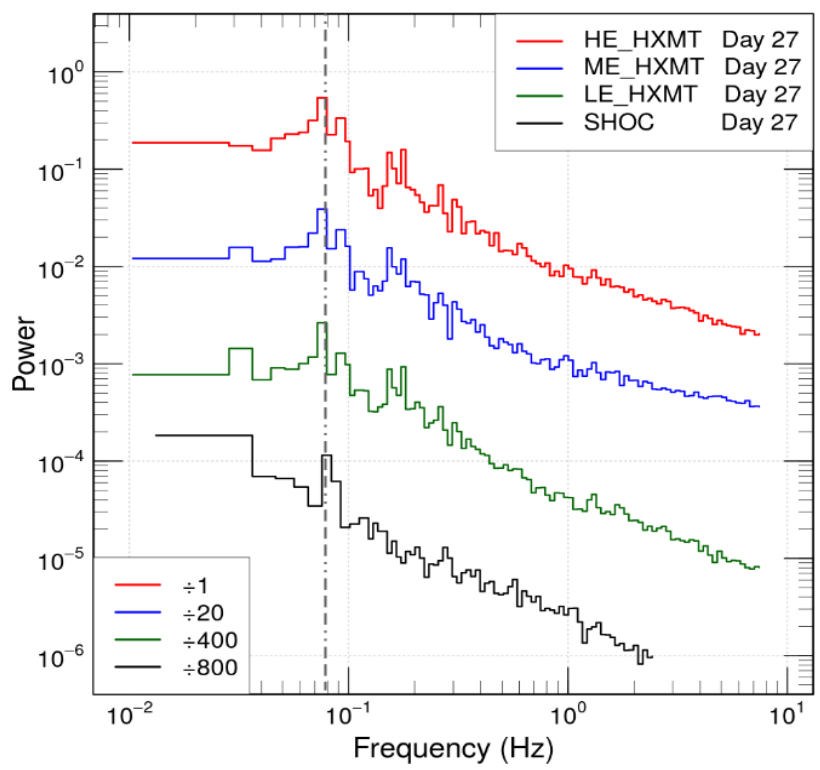

Figure 2. X-ray QPOs from Insight-HXMT LS power spectra in the HE, ME and LE bands (see box for colour coding), on day 27 (7 April) simultaneous with the SHOC optical QPO. The vertical dotted-dashed line is passing through the QPO values.

allow us to probe the emitting regions close to the central black hole by finding how different wavelengths lag one another within each system.

Our fast SAAO photometry was undertaken simultaneously in X-rays with NICER and Insight-HXMT, which resulted in the CrossCorrelation Functions (CCFs) presented in Figure 4. These were produced by splitting the light-curves into segments of equal length ( $30 \mathrm{~s})$, which were then 'pre-whitened' (i.e. had the linear trend re-

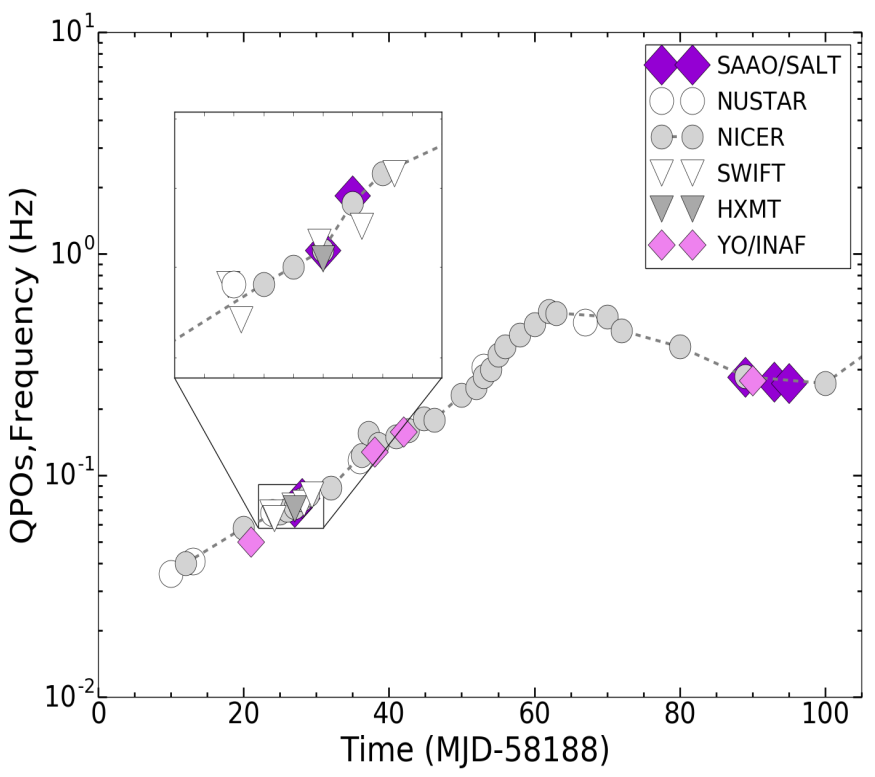

Figure 3. Temporal evolution of the optical and X-ray QPOs detected through the hard state of the J1820 outburst. The grey dotted line represents a polynomial fit to the Stiele \& Kong (2020) NICER QPO fundamental frequencies. See box (and text) for observatory identifications.

moved), and a CCF produced from each using the methodology of Venables \& Ripley (2002). The mean of the CCFs was then determined, and the standard error on each bin calculated.

SAAO observations were simultaneous with NICER on days 15 and 16, and with Insight-HXMT (LE, ME, HE bands combined) on day 27 . For days 15,16 , and 27 , we used 45,22 , and 47 segments (totalling 1350, 660, and $1410 \mathrm{~s}$ ) respectively.

\section{DISCUSSION}

\subsection{CCF Results}

The CCFs presented in Fig. 4 show some key features also seen in other BHXBs. For example, a sub-second optical lag has also been seen in XTE J1118+480 (Kanbach et al. 2001), GX 339-4 (Gandhi et al. 2008, 2010), and V404 Cyg (Gandhi et al. 2017). Additionally, a dramatic anti-correlation at negative lags has been seen in several sources, most significantly in Swift J1753.5-0127 (Durant et al. 2008) and, to a lesser extent, BW Cir (Pahari et al. 2017) and XTE J1118+480.

There are multiple ways to physically interpret these features, and they have been variously ascribed to such phenomena as a jet (Malzac et al. 2018) and a hot inner accretion flow (Veledina et al. 2011; Veledina et al. 2013a). It is currently unclear as to which phenomena are the most significant in $\mathrm{J} 1820$, and what its geometry is - see, for example, discussions on a changing coronal height (Kara et al. 2019), a moving inner disc radius (Buisson et al. 2019; Zdziarski et al. 2021a; De Marco et al. 2021), the presence and strength of a jet (Bright et al. 2020; Tetarenko et al. 2021), and some combination of jet and hot flow components (Veledina et al. 2019; Paice et al. 2021). There has also been a suggestion (Poutanen et al. 2021) that the $\mathrm{BH}$ spin and orbital plane are mis-aligned by at least $40^{\circ}$.

What do our CCFs show? One change between days 15 and 27 


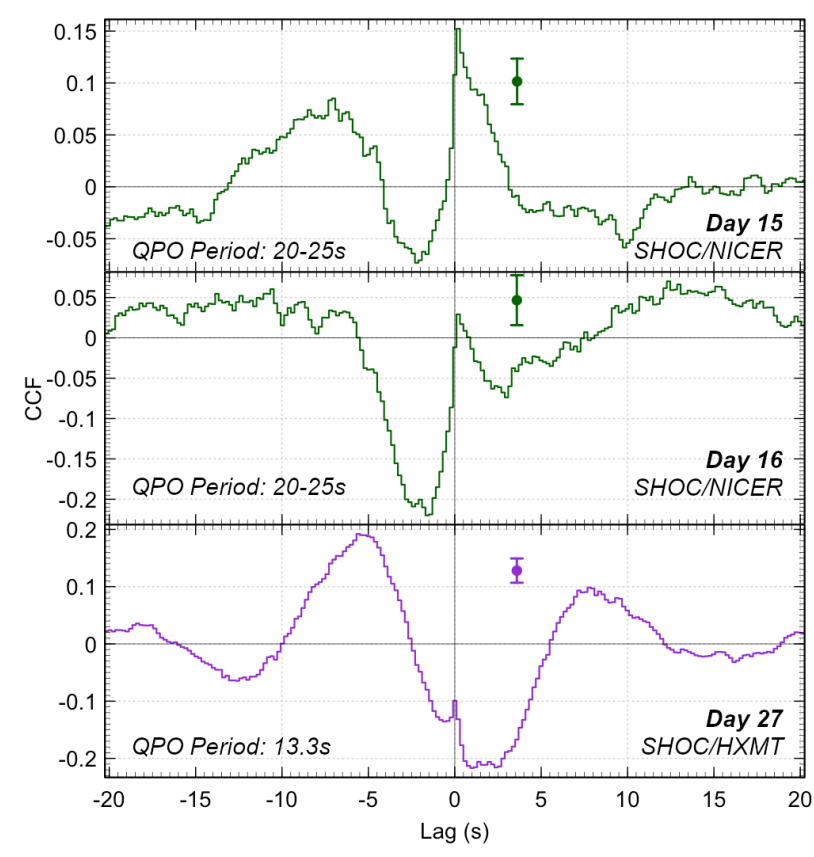

Figure 4. Optical/X-ray CCFs for the SAAO-SHOC vs NICER data obtained on days 15 (top) and 16 (middle) (dark green), and SAAO-SHOC vs InsightHXMT on day 27 (bottom, dark violet). Each CCF is made from the average of 30 s segments, with a representative standard error bar shown. Note the precognition dip present in days 15 and 16, which evolves into a broad anticorrelation by day 27 . Also note the sub-second peak at positive lags, which changes in shape over time.

is the reduction in significance of the sub-second lag. This was also seen in Paice et al. (2021) where it was ascribed to the jet decreasing in significance; these results put constraints on the timescale over which this occurred.

Our data from day 27 show a unique feature in J1820 that has not been seen as strongly in other BHXBs; a broad anti-correlation that straddles zero lag, rather than just at negative lags. Only GX 339-4 has shown something similar, and at much lower significance (Gandhi et al. 2008). Our data also show that this feature was still developing on day 16.

Another key feature is the QPO mentioned in Section 3.1, which can be seen clearly in the CCF on day 27. At this point in the outburst, the QPO has a frequency of around $0.075 \mathrm{~Hz}$, which corresponds to a period of $13.3 \mathrm{~s}$, which is roughly the distance between the negative correlation peak at $-5 \mathrm{~s}$ and the positive correlation peak at $+7 \mathrm{~s}$. This effect, of the QPO being visible in the CCF with the positive peak just over $\pi$ radians away, was also seen by Paice et al. (2021) who showed that it continued much later into the outburst (from day 37 up to at least day 88); the same work also showed the QPO to be strongly coherent. It is possible that the broad anti-correlation at positive lags could be at least partly due to the QPO, a possibility backed up by the QPO becoming more significant between our first and last CCFs (days 15 and 27; see Fig. 3, as well as Stiele \& Kong 2020, Fig. 8).

\subsection{Optical and X-ray QPOs}

$\mathrm{X}$-ray QPOs in the $\mathrm{mHz}$ frequency range have been previously detected from $\mathrm{J} 1820$ and it is exceptional to observe type-C QPOs below $1 \mathrm{~Hz}$ for large parts of a BHXB outburst (Stiele \& Kong 2020).

What is more remarkable in $\mathrm{J} 1820$ is that our optical photometry not only confirms the presence of optical QPOs in the frequency range of $\sim 0.04 \mathrm{~Hz}$ to $0.3 \mathrm{~Hz}$ from the beginning of outburst to the time of the state transition, but it also shows how well the optical and X-ray QPOs track each other throughout this time (Figure 3). We therefore have the following key properties that must be explained by any physical model for J1820:

- almost perfect tracking of the optical and X-ray QPOs throughout the hard state, across five orders of magnitude in photon energy, right up to the state transition itself,

- optical QPO exhibits no detectable harmonics, whereas the Xray QPO does, suggesting the optical emitting mechanism is more sinusoidal compared to the X-ray,

- optical QPO fractional rms $(\sim 3 \%)$ is smaller than that in X-rays $(\sim 10 \%)$,

- apart from the QPOs, the power spectra of both bands can be adequately fitted with two Lorentzians,

- Paice et al. (2019) have already shown with their HiPERCAMNICER campaign on J1820 that there is a $\sim 165 \mathrm{~ms}$ delay between the $\mathrm{X}$-ray and optical variations, together with a second, broader anticorrelated component on timescales of a few seconds. This is what we also observe.

- the restricted optical/Insight-HXMT power spectrum analysis shows that the optical is as "sharp" a feature as the X-ray.

Taken together, these properties indicate that the X-ray and optical QPO-emitting regions must be very close (within $\sim 0.1$ lt-sec), so there is no possibility of reprocessing at the outer disc or donor star driving the optical QPO. The data are consistent with the presence of two components dominating the variability.

We note that the already known X-ray/optical delay in J1820 is actually very similar to that seen in V404 Cyg (Gandhi et al. 2017), and so we should consider the scenario shown in their Figure 3. To this we can add the production of both X-ray and optical QPOs by making the jet precess at the QPO frequency, as proposed by Ma et al. (2021). In their model, the jet precession can well explain the large soft phase lag and the energy-related behaviour of the J1820 X-ray QPO from 1-250 keV. Furthermore, an optical QPO with a centroid frequency similar to that seen in X-rays is also expected. This has been suggested by García et al. (2021) in accounting for the X-ray spectral timing properties of the QPOs in MAXI J1348-630, and is based on the MHD simulations of relativistic, precessing jets by Liska et al. (2018), as shown in their Figure 2.

Additionally, if the X-ray base was more compact, then the precession would produce a more "square" wave, whereas the optical emitting region, higher in the jet, might be more smoothed out, explaining the different harmonic structure that we observe. Moreover, if the top of the jet is less curved, the fractional rms of the optical QPO should be smaller than the X-ray QPO, as the velocity modulation seen by the observer is relatively weak. Based on the model of Ma et al. (2021), the optical QPO rms of 3\% would then suggest that the optical jet is faster than $0.8 c$ (see Figure 5) - if we use the opening angles from the radio jet (Zdziarski et al. 2021a,b; Tetarenko et al. 2021), and considering that the jet contributes about half the optical emission (Shidatsu et al. 2018); the phase lag of $\pi$ indicates that the jet is curved, with the phase difference between the optical and X-ray emitting regions $\sim 180^{\circ}$.

The internal shock model of Malzac et al. (2018) can reproduce the $\sim 150 \mathrm{~ms}$ optical lag, as well as the anti-correlated variability through variations in Doppler-boosted jet emission. The properties of the precessing jet are driven by processes in the inner disc, and, as this moves inwards as the transition approaches, it must increase the QPO frequency. Furthermore, it will raise the X-ray base (De Marco et al. 2021), providing the raised X-ray emitting region required 


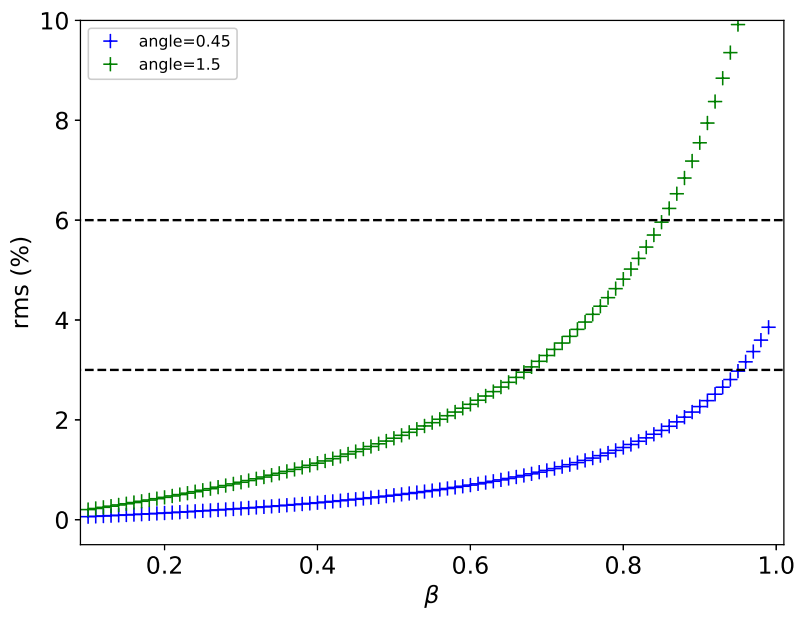

Figure 5. Relation between QPO rms and $\beta\left(=v_{\text {jet }} / c\right)$ for opening angles of $\phi=1.5^{\circ}$ (green, Zdziarski et al. (2021a)) and $\phi=0.45^{\circ}$ (blue, Tetarenko et al. (2021)). The lower horizontal dashed line indicates the original QPO rms of 3\%, while the upper line indicates the QPO rms of $6 \%$ from the jet when considering the contribution of the disk emission to the optical flux (Shidatsu et al. 2018).

to explain the optical "superhump" (Paper 1) from the outer disc regions.

While the above is a plausible self-consistent description, it does not rule out contributions from other mechanisms. In particular, the hot flow precession model of Veledina et al. (2013b) predicts optical and X-ray variations that are out-of-phase with each other at inclination angles of about $60^{\circ}$ (similar to J1820). But the hot flow model cannot, by itself, produce the sharp $150 \mathrm{~ms}$ optical lag. So a combination of a central hot flow producing the QPOs and an inner jet producing the $\sim 150 \mathrm{~ms}$ lag also appears to be viable.

\section{DATA AVAILABILITY}

The optical and X-ray data underlying this article are available from the following archives:

- SALT/SAAO - http://cloudcape.saao.ac.za/index.php/f/1009821

- NICER - https://heasarc.gsfc.nasa.gov/docs/archive.html

- Swift - https://www.swift.ac.uk/archive/

- Insight-HXMT - http://hxmtweb.ihep.ac.cn/

\section{ACKNOWLEDGEMENTS}

The SALT observations were obtained under the SALT Large Science Programme on transients (2018-2-LSP-001; PI: DAHB) which is also supported by Poland under grant no. MNiSW DIR/WK/2016/07. DAHB and SBP acknowledge research support from the National Research Foundation. JPL was supported in part by a grant from the French Space Agency CNES. L.T. acknowledge research support from the National Natural Science Foundation of China.
De Marco B., Zdziarski A. A., Ponti G., Migliori G., Belloni T. M., Segovia Otero A., Dziełak M., Lai E. V., 2021, arXiv e-prints, p. arXiv:2102.07811

Denisenko D., 2018, The Astronomer's Telegram, 11400, 1

Durant M., et al., 2008, ApJ Letters, 682, L45

Gandhi P., et al., 2008, MNRAS, 390, L29

Gandhi P., et al., 2010, MNRAS, 407, 2166

Gandhi P., et al., 2017, Nature Astronomy, 1, 859

García F., Méndez M., Karpouzas K., Belloni T., Zhang L., Altamirano D., 2021, MNRAS, 501, 3173

Kanbach G., et al., 2001, Nature, 414, 180

Kara E., et al., 2019, Nature, 565, 198

Kawamuro T., et al., 2018, The Astronomer's Telegram, 11399

Liska M., Hesp C., Tchekhovskoy A., Ingram A., van der Klis M., Markoff S., 2018, MNRAS, 474, L81

Ma X., et al., 2021, Nature Astronomy, 5, 94

Malzac J., et al., 2018, MNRAS, 480, 2054

Mereminskiy I. A., Grebenev S. A., Molkov S. V., Zaznobin I. A., Khorunzhev G. A., Burenin R. A., Eselevich M. V., 2018, The Astronomer's Telegram, 11488

Motta S., Muñoz-Darias T., Casella P., Belloni T., Homan J., 2011, MNRAS, 418,2292

Mudambi S. P., Maqbool B., Misra R., Hebbar S., Yadav J. S., Gudennavar S. B., S. G. B., 2020, ApJ, 889, L17

Muñoz-Darias T., Motta S., Pawar D., Belloni T. M., Campana S., Bhattacharya D., 2010, MNRAS Letters, 404, L94

Pahari M., Gandhi P., Charles P. A., Kotze M. M., Altamirano D., Misra R., 2017, MNRAS, 469, 193

Paice J. A., et al., 2019, MNRAS, 490, L62

Paice J. A., et al., 2021, MNRAS, 505, 3452

Pottschmidt K., et al., 2003, A\&A, 407, 1039

Poutanen J., et al., 2021, arXiv e-prints, p. arXiv:2109.07511

Shidatsu M., et al., 2018, ApJ, 868, 54

Stiele H., Kong A. K. H., 2020, ApJ, 889, 142

Tetarenko A. J., et al., 2021, MNRAS,

Thomas J. K., Charles P. A., Buckley D. A. H., Kotze M. M., Lasota J.-P., Potter S. B., Steiner J. F., Paice J. A., 2021, MNRAS,

VanderPlas J. T., Ivezić Ž., 2015, ApJ, 812, 18

Veledina A., Poutanen J., Vurm I., 2011, ApJ Letters, 737, L17

Veledina A., Poutanen J., Vurm I., 2013a, MNRAS, 430, 3196

Veledina A., Poutanen J., Ingram A., 2013b, ApJ, 778, 165

Veledina et al., 2019, A\&A, 623, A75

Venables W. N., Ripley B. D., 2002, Modern Applied Statistics with S, Fourth Edition. Springer, doi:10.1007/978-0-387-21706-2

Yu W., Zhang J., Yan Z., Wang X., Bai J., 2018, The Astronomer's Telegram, 11510

Zampieri L., et al., 2019, Contributions of the Astronomical Observatory Skalnate Pleso, 49, 85

Zdziarski A. A., Dzielak M. A., De Marco B., Szanecki M., Niedzwiecki A., 2021a, arXiv e-prints, p. arXiv:2101.04482

Zdziarski A. A., Tetarenko A. J., Sikora M., 2021b, arXiv e-prints, p. arXiv:2108.10929

\section{REFERENCES}

Bright J. S., et al., 2020, Nature Astronomy, 4, 697

Buisson D. J. K., et al., 2019, MNRAS, 490, 1350

Coppejans R., et al., 2013, PASP, 125, 976 